\title{
Economic model for the evaluation of $3 D$ food printing
}

\author{
Luca Dabbene, Lucia Ramundo, Sergio Terzi \\ Department of Management, Economics and Industrial Engineering \\ Politecnico di Milano \\ Milan, Italy \\ luca1.dabbene@,mail.polimi.it, lucia.ramundo@polimi.it, Sergio.terzi@polimi.it
}

\begin{abstract}
The food sector is one of the most relevant at global level and nowadays it faces new challenges coming from the emerging trends of personalized nutrition, food customization and by the growing number of people with food allergies and intolerances. Additive Food Manufacturing (AFM), a new technology having a considerable growth of interest in the last years by researchers and food experts, seems to be a promising solution to address those issues. Nowadays the academic studies on 3D food printing, another popular name of AFM, are still under development and they are focalized on technological and technical elements of the production process. The studies leave outside in most cases practical implications that the adoption of the technology will have from an economic, social or sustainability perspective. This paper has the objective of develop an economic model to evaluate the introduction of the additive manufacturing technology in the food market. More in detail the model describes possible changes due to the adoption of 3D food printing in relation to small food business, like restaurants and confectionery. The study is focalized on the economic aspect, analyzing also effect and changes that the new technology will have along the supply chain.
\end{abstract}

Keywords-3D food printing; Additive Food Manufacturing; AFM; economic model

\section{INTRODUCTION}

This paper aim is to study Additive Food Manufacturing (AFM), most commonly known as 3D food printing, possible applications in a real world business context. Its main objective is presenting a model for the evaluation of the introduction of 3D printing technology in the food sector; this model wants to be a roadmap to help firms and practitioners to effectively drive the implementation process. The purpose of the model is to investigate how the possible adoption of 3D food printing will change the landscape of the food sector, studying the effect of the technology not only on the firms that directly adopt it, but also on the players upstream and downstream in the supply chain. The analysis of the business context shows three possible scenarios of application: home users, small-batch production and industrial scale production [13][15]. At the current status, anyway, the small batch production, meaning $\mathrm{B} 2 \mathrm{C}$ companies, like restaurants, bakeries and similar activities, seems to be the most promising one for the adoption of the technology. In the following section the paper discusses how the study was performed, the main findings and the possible future steps of the research. The first section of the paper gives an overview about the scientific literature existing at the moment on the $3 \mathrm{D}$ food printing topic, its actual direction, and analyzes the possible gaps and lacks in the stream of studies. The second section gives an overview about Additive Food Manufacturing technology and then presents the methodologies used in the study to develop and validate the model. The third section presents the main findings of the study, including a detailed discussion of the model structure, its purpose and validation. The fourth and last section of the paper discloses the achieved objectives and the possible future directions to improve the conducted research.

\section{RELATION TO EXISTING THEORIES AND WORK}

Additive Manufacturing (AM) also known as "3D printing" is a term that includes a broad range of manufacturing process technologies that are emerging to offer the prospect of ondemand, mass personalization, with more localized, flexible and sustainable production [1] [6]. One of the big advantages of AM is the opportunity to produce customized pieces, even in small quantity, at low price compared to traditional processes [18][20][21][32]. AM applied to the production of food can be a solution to effectively address the new trends arising in the market. This technology is called Additive Food Manufacturing (AFM) or 3D food printing and it is still under development [8][14][15][20]. Several production techniques have been developed during years; the main are extrusion based process, inkjet printing process and powder binding deposition [7][18][20]. Extrusion process is the most promising and developed, because all the research studies use it as the printing method for the study of process and material improvement thanks to its advantages: higher degree of freedom in the design of pieces and a wider array of foods suitable for the production process [5] [10][12][17][19][22]. The purpose of AFM is not to concentrate manufacturing processes of products in a single step, but it is associated with the design of food with new textures and potentially enhanced nutritional value [7][16][32]. The real potentiality of 3D food printing is the opportunity to produce $3 \mathrm{D}$ customized- 
designed food objects with higher production efficiency and reducing manufacturing cost for customized food products fabrication [15][21]. AM in the food sector is carried by the possibility to design complex structures which are not possible to design manually by an artisan, and through the use of $3 \mathrm{D}$ food printing to answer specific nutrition-related problems [21][22]. However, nowadays 3D food printing is more a novelty linked to small niche market than an industry changer: restaurant chains and packaged-food big firms seem unlikely to convert entire production lines to print most of what customers eat and buy [2][13]. This paper wants to address the research questions of how AFM will be adopted in real world application and the effects that it will have on the context in which it will be implemented. The analysis of the existing scientific literature about the state of the art of the academic research on $3 \mathrm{D}$ food printing shows that the basics of the technology, its functioning and the different production processes are well known and discussed in literature [7][18][20]. In 2014/2015 the research studies were mainly on two topics: the possible advantages in the implementation of this technology, issues and field of application with future direction and the study of material properties and process parameters needed to optimize the printing process. However, in 2016/2017 the research studies leave the first theme of discussion to focus on the second topic: the optimization of the process [4][11][12]. This new interest seems to be related to the need of improving the general performances of speed, reliability and final products quality and enlarges the range of materials suitable for $3 \mathrm{D}$ food printing [22]. This review of existing literature shows the lack of studies on the possible business implementation of $3 \mathrm{D}$ food printing and its evaluation in the food industry from economic, social, and environmental point of view. The food industry, worth $\$ 5$ trillion represent 10 percent of global consumer spending, 40 percent of employment, and 30 percent of greenhouse-gas emissions[25]. Due to its economic relevance, understanding the effects, that AFM will have on it, will help to foresee future direction of development and possible economic impacts. Some of the biggest trends that is expected to influence the sector in the future are related to customization on individual preferences or on individual needs [20][21]. The first is related to the offer of new foods to customers and the real challenge of this trend is to create individual personalized foods, in terms of shape, color, flavor and, generally, of all the element that compose the eating experience [20]. The second is related to personalized nutrition, so adapt the nutritional values of foods to individual needs [14]. In the last few years nutritional experts have criticized the traditional approach [24][25] to nutritional advice, as it fails to consider individual's surroundings, habits, and DNA, along with many other complex contributing factors there is the need to find new method to address this issue. Companies are starting to offer personalization services, and also some big player in the food industry are experimenting into this space. One of the biggest problem to satisfy these two trends is related to the difficulty to create personalized products at small batches and with low costs with actual mass production process used in the food sector by big firms. Both trends are pushing the industry towards solutions that enable the production of customized products at low volumes with acceptable costs, that will be translated in affordable prices for final customers. On the other hand customized and personalized food is the core business of small batch production companies, such as confectionery shops, bakeries, restaurants and similar activities Considering the above scenario, the technology looks to be more suitable of adoption by those last small companies, than a mass production industry. In literature, for instance, it is already available one research study [9] that analyzes how the adoption of 3D food printing could change the supply chain of the chocolate sector and the economic effect of this technological implementation. Anyway, a more general study on the real-world effect of the implementation of $3 \mathrm{D}$ food printing is still missing and the research presented in this paper is an attempt to cover that big gap found in literature.

\section{RESEARCH/TECHNOLOGY/INNOVATION APPROACH}

The research study has been conducted, as already stated above, starting from a deep scientific literature analysis of the state of the art about 3D food printing and the possible adoption of the technology in real world context. Other than scientific papers, also additional sources of information have been used such as web sites, market and positioning reports. Interviews to researchers, to food players and to members of $3 \mathrm{D}$ food printing firms have been performed with the purpose of collecting info, verifying acquired knowledge and validate the research results. The "3D Food Printing Conference" in Venlo on $17^{\text {th }}$ June 2017 , has been used as an additional source of information, subsequently used in the research to develop the model, and to understand the possible future dynamics of the market. Here researchers and business managers were contacted and then subsequently employed as experts in the validation phase in order to gather as much information as possible directly from the industry.

The system thinking method has been the chosen methodology to develop the economic model. This seems to be a valid solution to the research question as it is an approach that emphasizes the relationships among the system's parts rather than the parts themselves. The systems thinking assumes that the understanding of a system has to take into account an analysis of its individual constituents as well as of their inter-linkages and relationships[2]. In systems thinking, cause and effect do not always follow a straight line whose end is set apart from its beginning. Instead, actions can be circular; their effects fold back to become a cause[3]. Another relevant feature of systems thinking is that it considers human actions. British professor Ralph Stacey describes this aspect [2]: "Systems thinking is a holistic way of thinking that respects profound interconnectedness and... puts people, with their different beliefs, purposes, evaluations and conflicts, at the center of its concerns". In this case there is a starting hypothesis that will be the center of the model, and the 
objective was to understand, analyze and represent how this hypothesis will influence the other elements of the system. These elements, when modified will affects other parts of the system, leading at the end to a more or less deep modification of the whole environment considered.

The current status of the 3Dfood printing market adoption is at the very beginning, since, as stated above, the AFM companies are still experimenting on the technology issues and there are not public market data available. This means it is not possible to evaluate the model using real and reliable data and then quantitative methods. The validation of the model has then been carried out through a qualitative validation method through sector experts. More in detail, experts include 3Dprinters companies, small batch production food companies and food enterprises. A double check methodology has been used. In a first round, the model has been submitted to experts. All the feedbacks and information have been collected and used to improve the model. When the new draft of the model has been completed, a second round of validation has been done. The model, in fact, has been sent back to the same experts to receive additional feedbacks about further improvement or correction.

\section{FINDINGS}

This section presents the developed model and discusses the main findings. The model is presented in figure 1 .

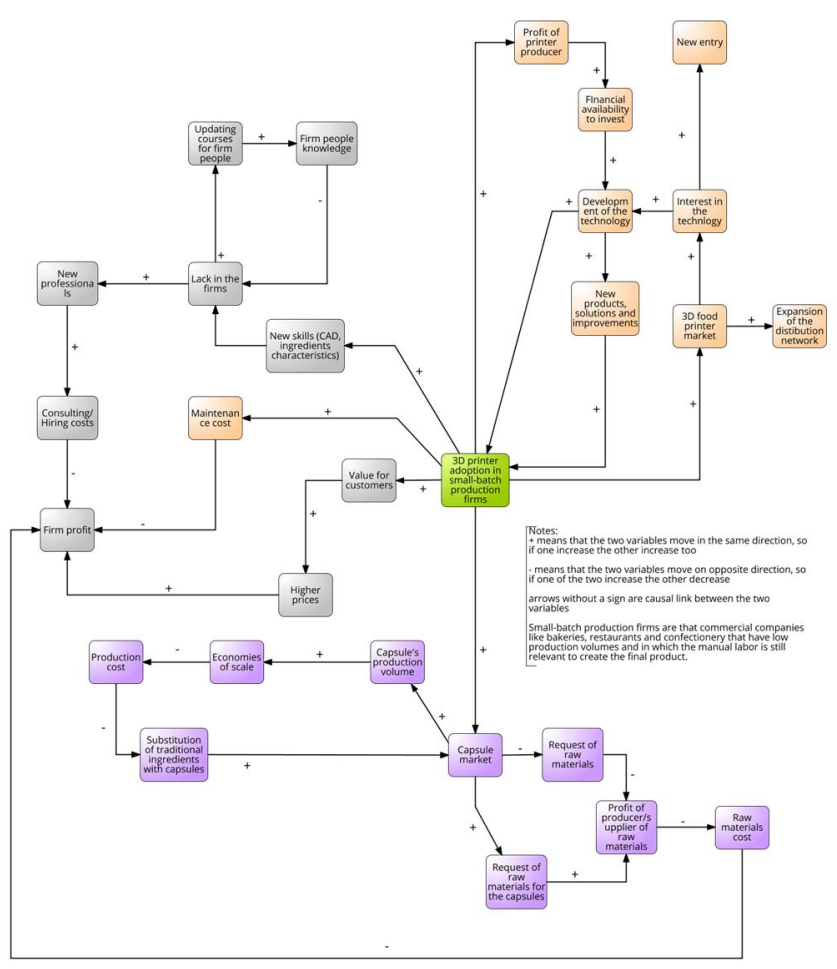

Fig. 1 The AFM adoption economic model

The economic model, as stated above, has been developed through the system thinking methodology and shows the changes that will arise in the small-batch production field of the food sector after the adoption of 3D food printing, in order to help early adopters to have the whole picture scenario needed to evaluate the adoption of the AFM in their business The model is composed of three main sections: raw material suppliers, new skills and printer producers. All the three sections born from the initial hypothesis of the adoption of $3 \mathrm{D}$ food printing in the small batch production sector and each of them analyzes a different aspect of the activities performed by small-batch production firms that will be influenced by $3 \mathrm{D}$ food printing adoption. Each section is built in order to analyze the effect of the adoption not only on the small-batch production sector but also on the other element of the supply chain. The three sections are designed also to understand possible feedbacks effects that will happen after the adoption of the technology, meaning how the changes happening to the supply chain will influence the adoption itself. Here follows the analysis of each section of the model in more detail.

The first section is the raw material suppliers part, starting from the analysis of the market for food capsules, as showed in figure 2. These capsules contain the raw materials, so the ingredients of food printed products, that will be pre-worked to have the right set of characteristics to be printed.

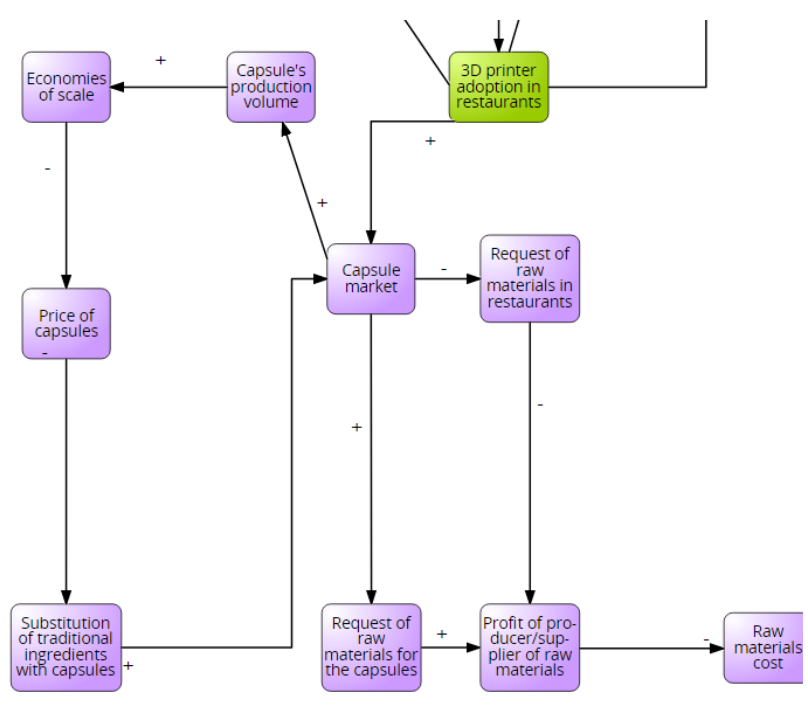

Fig. 2 The row material section of the economic model

These capsules have a higher cost compared to the same kind of ingredients in their rough form, due to the additional activities performed on the raw materials. Nowadays, due to the extremely low number of 3D food printers used around the world, this market almost doesn't exists, but it can be assumed that with the adoption of 3D food printing technology in the small-batch production food sector the request for the capsules increase significantly, creating a market and giving the possibility to study the consequences on the rest of the supply chain. In this case the focus of the model is on the changes that the producer of capsules will face and, on the changes, that traditional supplier of that 
market may face. The analysis considers a generic capsules and ingredients market, to see how these two elements influence each other and how they react to the new situation, without focusing on a specific kind of capsules or ingredients. The first effect faced by producers of capsules, when the adoption of 3D printing technology starts and capsule market born, will be an increase of the demand for their products. With the increase in demand and the following increase in the production volumes they could face the rise of economies of scale that will reduce the production cost of capsules. The economies of scale could arrive from the adoption of more advanced manufacturing techniques or machines that enable the production of higher volume of products at a lower unitary cost in respect to the ones actually employed. This will be true in both the cases were capsules producers make themselves all the capsule or use external suppliers for some components. With the reduction of the capsules price, $3 \mathrm{D}$ printing technology can start to become an interesting alternative also in the direction of substituting traditional production method other than for create new, complex and customized products, that is currently its greater advantage and the main reason for its adoption at the beginning. More the cost of capsules becomes closer to that of the raw ingredients more this phenomenon will grow, leading to a higher use of capsules, consequently to an increase of the demand that creates a positive loop for the capsules market for all the reasons discussed above. The last point to consider is about the supply chain of raw materials and the effect that 3D food printing could have on the producers of traditional ingredients. As the traditional ingredients will be used also in the creation process of the filling for food capsules here, there will be two possible alternatives for the supply chain. The two supply chains proceed in the same way until the point in which raw materials instead of going directly at small-batch production firms through wholesale suppliers, could make an additional step passing from capsule producer companies. This will introduce the new raw material supplier area into the supply chain. After the adoption of 3D food printing both path will remain valid as long traditional ingredients continue to be used in activities of small-batch production firms, but considering a longer period of time the possible evolution of the employment and adoption of the technology could led to different effects on traditional ingredient producers. From the supplier's side, at a first sight, the increase in demand for raw materials for capsules will result in an increase of profit. Actually this can be a misleading conclusion, because to determine the real impact of the adoption of 3D food printing on that variable, several aspects have to be taken into account. First, the adoption of the new technology and the following increase in the use of capsules may lead to a decrease in the use and so in the demand for traditional ingredients. Since the producers and suppliers of raw materials are the same in both cases of capsules and traditional ingredients they may experience both an increase of demand coming from the capsule market and at the same time a decrease of demand coming from the old market of traditional ingredients. Nowadays, it is difficult to foresee the value of these changes in demand and the effect that their balancing has on the overall demand for the raw material market.

The second aspect to discuss about the model is related to new skills required by small batch production firms after the adoption of the technology. This part of the model is reported in figure 3.

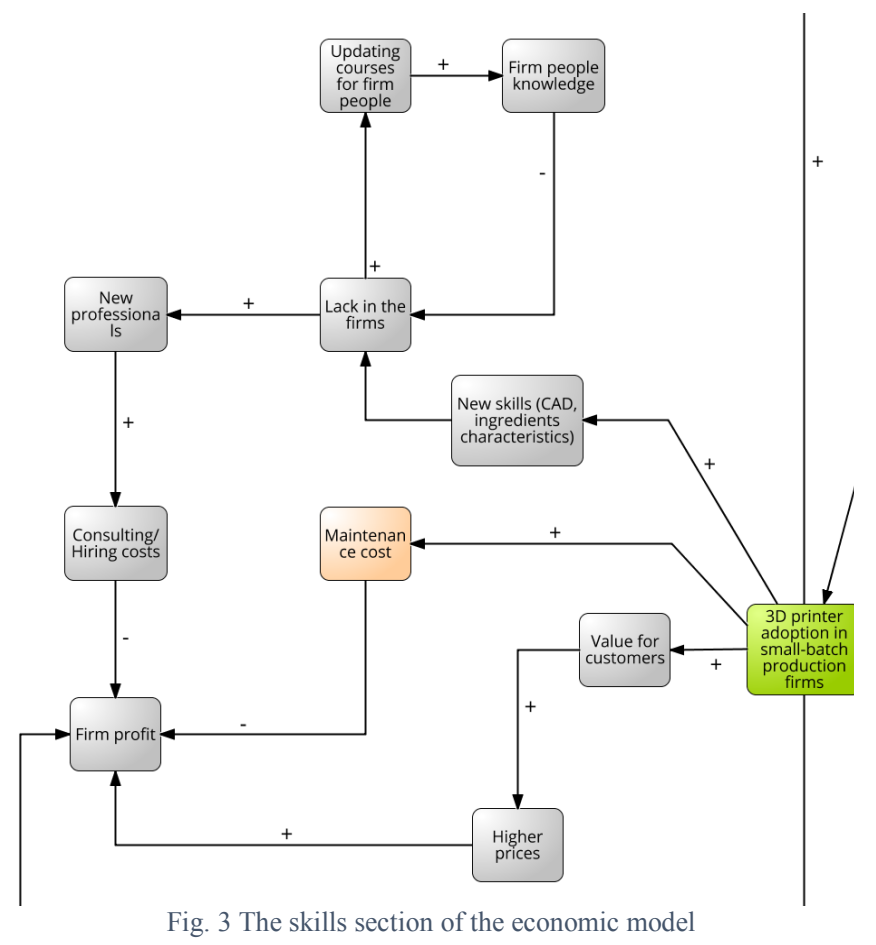

Although 3D printers are easy to use and with a short training and some trials people are able to use them without problem as a basic tool, they require some specific knowledge to exploit their full potentiality. This knowledge is of two main type: computer aided design (CAD) knowledge and food characteristics knowledge. CAD knowledge is fundamental to create the $3 \mathrm{D}$ models of the objects, that transferred to the printers are the starting point for the production process. Food characteristics don't refer only to the knowledge and ability to create possible combination of ingredients and the way to treat them to have good taste, consistencies and in general required properties for the eating experience of customers. They include also knowledge of the material properties that may influence the printing process and that need to be taken into account when a new piece will be designed to assure its feasibility, stability and safety. In addition, specific skills are needed to create optimized capsules, and this increase the difficulty for firms to develop the fills on their own. In the small-batch production sector there is a big lack of the two kinds of knowledge just discussed. Regarding food characteristics, small-batch production firms already have some knowledge on foods and ingredients and have capabilities to manage them in a proper way. This can help the process, but is not enough to be able to use 3D food printers appropriately. For CAD knowledge the actual situation is even worse, because nowadays these skills are 
completely absent in small-batch production firms, but can be easily developed in the future. The first solution for smallbatch production firms to solve the skills problem and cover the gap is to call on practitioners that already possess these kinds of knowledge. On the market nowadays, CAD skills are common, and firms will easily find a CAD designer for their needs. From now on we call food experts the food technologists that possess specific knowledge on food characteristics that are needed and that are not common and even less easily find on the labor market. These experts in most of the cases are researchers that work in universities or similar institutes and for small companies can be difficult to reach them and ask for consultancy or to try to hire them. As at the beginning of the adoption process this is the only way to find the kinds of expertise needed to exploit all the opportunity offered by the new technology. Considering a longer period of time firm may start to think about the possibility to internally develop these skills. At the end the need for these professional roles, completely new for the sector will be translated in new employment opportunities. The third part of the model is about the new business opportunity enabled by the technology, as shown in figure 4 .



Fig. 4 The technological section of the economic model

The adoption of 3D printing in small-batch production food sector gives the opportunity to firms to create new type of offers with higher values for final customers. The increase in the offer's value will come mainly from the new customization opportunities that the technology enables. Nowadays the creation of food and dishes with strange or personalized shape requires a lot of expertise, time and manual labor making it very costly and difficult to obtain, and not all the companies offer such services. With 3D food printing every firm will be able to offer a high level of customization possibility to its clients, at a reduced cost compared to actual situation. At the end all these factors will impact on the profit of firms. These impacts may regard costs, savings and new possibilities of profit. Here comes the opportunity for the producers of 3D food printers. The market for $3 \mathrm{D}$ food printers is currently small and it is still in development phase. The players are showcasing their products and they expect to commercialize them in the market soon. Despite the attempt made by these companies to spread knowledge and information and to advertise people and firms about 3D food printing, the majority of food organization and general people are still unconscious about the existence of the technology. This can be caused by the novelty, that makes difficult to reach the larger part of the audience, but also from some mistakes or wrong choice that printer producers make in the way they publicize their products. Under the initial hypothesis of the adoption of 3D food printing it is possible to expect that these issues will be overcome and that the demand and so the market for 3D food printers will start to grow. With this growth of the market, the interest for this new technology will start to grow as well. In the scientific community there are already a lot of expectations around this technology and the interest of researchers and scholars is high. The growth of the market and the adoption of 3D food printing by business firms may also increase the interest in the technology by firms that operate in the food sector. They might start to check the technology and its potentiality and the advantages offered, in a perspective of a possible adoption. The other element to consider is that the increase in profits means a higher financial availability, that can be reinvested in $R \& D$ activities by firms to further innovate. This can increase the research of scientific community and firms about the $3 \mathrm{D}$ printing technology in the food sector, providing new solutions, improvements and innovations.

The economic model about the evaluation of AFM, just discussed, has been validated through interviews and question to experts in the 3D food printing sector and smallbatch production food sector. For this last category of experts, different restaurant and confectionery owners have been contacted. The purpose of interview to those experts was to get information about the real use that firms in the smallbatch production food field will make of the technology, their concerns about it and to have a comparison with traditional production methods currently used. The model has been submitted to them through e-mails and direct interviews, explaining the model's objective, the way in which it was built with all the assumption, hypothesis and information used to give a complete picture of the research. After this explanation phase, it has been asked them if the assumption and hypothesis are correct, as they are the basics of the model and have impact on all the following pieces of the analyses. The most critical hypothesis is the one related to the need for firm of using pre-filled capsules instead of creating themselves the fills, as it influences the part of the model 
related to raw materials. Another critical hypothesis is that one about the skills required by small batch production food firms. Both these assumptions were developed according to the literature review and the participation to the $3 \mathrm{D}$ printing conference in Venlo. The experts' answers have confirmed both of those assumptions and it has been possible to proceed and validate the whole model. The validation has been made with both categories of experts, technology producers and small batch production food companies, as each of them can give different feedbacks and suggestion, according to their different perspective on the issues addressed in the model. This has also helped in having the best and complete picture of the supply chain and of the impacts the hypothesis can have on it. During these interviews several feedbacks have been collected on different elements that weren't previously considered. Those feedbacks have been subsequently included in the final version of the model. At the end the model has been considered correct by all the interviewed experts, so it can be possible to assess its validity.

\section{CONCLUSION}

The developed model has been demonstrated to be an instrument to understand how the small-batch production food supply chain will be modified by the adoption of 3D food printing technology. This model can help 3D printer producers to understand what direction they have to follow to speed up and improve the adoption of the technology and it can be used by small-batch production food firms for understanding how they can effectively use 3D food printing, both to open new business opportunities and enhance existing ones. Moreover, the adoption of 3D food printing could affect significantly the supply chain of ingredients, until the raw material suppliers and the labor market as small-batch production firms will require new kinds of expertise. These findings have been achieved thanks also to the methodology to which the model was build: System thinking methodology. This has enabled, in fact, to consider all the possible sideeffects that the adoption of 3D food printing will have on the different players in the sector and on the different element of the environment. The drawbacks of this approach is that it is a qualitative method, so it is not useful to perform deeper economic analysis that could give a better understanding of the phenomena. It has not been possible to perform a quantitative analysis during both the development and the validation phase of the model also because data on the market for printers and food capsules are not commercialized yet or extremely difficult to find. This problem comes from the extremely small, if not inexistent, market for these kinds of products that consequently create a lack in information and data availability. Retrieve data and information on their demand and production cost is impossible using normal researching methods. The few technology producers working in this fields and the few identified end users are not willing to release them. The reluctance to give outside these sensible data can derive from the novelty of the market, in which be a step-ahead of other firms can have a big impact as it gives a lot of advantages in the competition. Starting from this situation it is possible to affirm that a great improvement could be the assessment in a quantitative way of the economic effects of the adoption of 3D food printing, gathering data about the potential market of 3D food printers to understand the real extend of the adoption phenomena. Another analysis can be performed to understand why the technology is so little known and what actions could be put in place to make firms aware of its existence. There is also the need to assess the real use that small batch production food firms will make of the technology, studying first adoption use cases. Other relevant points to highlight are $3 \mathrm{D}$ food printing social impacts and sustainability, to fully understand the implication of its adoption. Talking about the social implications of the technology, firstly there is the need understand how many people are aware about the technology and the degree of acceptance that $3 \mathrm{D}$ food printing in general will have. As a complete new way of produce foods, it will may face some trouble at the beginning about the safety and healthy aspect of products [23]. In this direction some studies on what people think about this technology, their expectations and concern can be made to assess the readiness of the final markets for 3D food printers' products. The other potential advantage with a potential social impact, also linked with sustainability refers to the use of alternative ingredients. This issue is much more relevant in western countries as a lot of alternative ingredients come from sources considered strange and usually not edible like insects or seaweeds. Here there is the need to understand how much these new ingredients will be accepted by people, and which can be the action to take for pushing people towards the acceptance of these ingredients [15]. Another possible direction of study is related to sustainability, which refers to a wide range of topics, from the reduction in the consumption of resources to solving social and environmental problems [16][23]. 3D food printing theoretically has a lot of potentiality in addressing sustainability issues, but so far no quantitative study was performed. Some sustainability topics discussed in literature are related to new alternative ingredients and the opportunity to use them to solve the food scarcity problem. New ingredients like insects and algae have higher nutritional value and can be produced in high quantity with lower resources compared to traditional ingredients [22]. 3D food printing is highlighted as a possible solution to obtain food products attractive from the point of view of their look but created starting from these alternative ingredients that in western countries are usually considered disgusting [5][7]. With the increase in the global population, researchers say [8][16][21][23] that if we can use these alternative sources we can be able to satisfy the overall demand for foods, without further negative effect on the environment. Study in this direction can be made assessing the real savings enabled by the use of these resources and possible drawbacks. At the end a further development of the study can be the creation of two other models, studying changes that will arise in smallbatch production sector also from a social and from a sustainability point of view. 


\section{REFERENCES}

[1] ASTM, ASTM F2792-12a: Standard Terminology for Additive Manufacturing Technologies, ASTM International, West Conshohocken, PA, 2012.

[2] Karen L. Higgins (2013). Financial Whirlpools: A Systems Story of the Great Global Recession. Oxford: Academic Press.

[3] J. Mingers, L. White / European Journal of Operational Research 207 (2010) 1147-1161

[4] Derossi, Antonio \& Caporizzi, Rossella \& Azzollini, Domenico \& Severini, Carla. (2017). Application of 3D printing for customized food. A case on the development of a fruit-based snack for children. Journal of Food Engineering.

[5] Fan Yang, Min Zhang \& Bhesh Bhandari (2017) Recent development in $3 \mathrm{D}$ food printing, Critical Reviews in Food Science and Nutrition.

[6] Ford, Simon \& Mortara, Letizia \& Minshall, Tim. (2016). The Emergence of Additive Manufacturing: Introduction to the Special Issue. Technological Forecasting and Social Change.

[7] Godoi, Fernanda \& Prakash, Sangeeta \& R. Bhandari, Bhesh. (2016). 3d Printing Technologies Applied for Food Design: Status and Prospects. Journal of Food Engineering.

[8] Hanley, Bryan. (2016). Additive manufacturing in food and nutrition. Nutrition Bulletin.

[9] Jia, Fu \& Wang, Xiaofeng \& Mustafee, Nav \& Hao, Liang. (2015). Investigating the Feasibility of Supply Chain-Centric Business Models in 3D Chocolate Printing: A Simulation Study. Technological Forecasting and Social Change.

[10] Lanaro, Matthew \& P. Forrestal, David \& Scheurer, Stefan \& J. Slinger, Damien \& Liao, Sam \& Powell, Sean \& Woodruff, Maria. (2017). 3D printing complex chocolate objects: Platform design, optimization and evaluation. Journal of Food Engineering.

[11] Le Tohic, C., O'Sullivan, J.J., Drapala, K.P., Chartrin, V., Chan, T., Morrison, A.P., Kerry, J.P., Kelly, A.L., Effect of 3D printing on the structure and textural properties of processed cheese, Journal of Food Engineering (2017)

[12] Lille, Martina \& Nurmela, Asta \& Nordlund, Emilia \& MetsäKortelainen, Sini \& Sozer, Nesli. (2017). Applicability of protein and fiber-rich food materials in extrusion-based 3D printing. Journal of Food Engineering.

[13] Lipton, Jeffrey \& Cutler, Meredith \& Nigl, Franz \& Cohen, Dan \& Lipson, Hod. (2015). Additive Manufacturing for the Food Industry. Trends in Food Science \& Technology.

[14] Lipton, Jeffrey. (2017). Printable food: the technology and its application in human health. Current Opinion in Biotechnology.

[15] Liu, Zhenbin \& Zhang, Min \& Bhandari, Bhesh \& Wang, Yuchuan. (2017). 3D printing: Printing precision and application in food sector. Trends in Food Science \& Technology.

[16] Lupton, Deborah. (2017). 'Download to Delicious': Promissory Themes and Sociotechnical Imaginaries in Coverage of 3D Printed Food in Online News Sources. Futures.

[17] Pallottino F. \& Hakola L. \& Costa C. \& Antonucci F. \& Figorilli S. \& Seisto A. \& Menesatti P. (2016). Printing on Food or Food Printing: a Review. Food Bioprocess Technology.

[18] Pinna, Claudia \& Ramundo, Lucia \& Sisca, Francesco \& Angioletti, Cecilia \& Taisch, Marco \& Terzi, Sergio. (2016). Additive Manufacturing applications within Food industry: an actual overview and future opportunities.

[19] Severini, Carla \& Derossi, Antonio \& Azzollini, Domenico. (2016). Variables affecting the printability of foods: Preliminary tests on cereal-based products. Innovative Food Science \& Emerging Technologies.

[20] Sun, Jie \& Peng, Zhuo \& Zhou, Weibiao \& Fuh, J \& Hong, Geok-Soon \& Chiu, Annette. (2015). A Review on 3D Printing for Customized Food Fabrication. Procedia Manufacturing.

[21] Sun, Jie \& Zhou, Weibiao \& Dejian, Huang \& Fuh, J \& Hong, GeokSoon. (2015). An Overview of 3D Printing Technologies for Food Fabrication. Food and Bioprocess Technology.
[22] Sun, Jie \& Zhou, Weibiao \& Yan, Liangkun \& Huang, Dejian \& Lin Lien-ya. (2017). Extrusion-based food printing for digitalized food design and nutrition control. Journal of Food Engineering.

[23] Tran, 3D-Printed Food, 17 Minn. J.L. Sci. \& Tech. 855 (2016)

[24] www.forbes.com

[25] www.fao.org 